\title{
Food Intake Activity Detection Using an Artificial Neural Network
}

\author{
S. Päßler ${ }^{1,2, *}$, W.-J. Fischer ${ }^{1,2}$ \\ ${ }^{1}$ Fraunhofer Institute for Photonic Microsystems (IPMS), Dresden, Germany \\ ${ }^{2}$ Institute for Semicondurctors and Microsystems (IHM), TU Dresden, Dresden, Germany \\ *Sebastian.Paessler@ipms.fraunhofer.de
}

\begin{abstract}
The World Health Organization pointed out an increasing percentage of obese people in the past decades. It is the reason for many cases of cardiovascular diseases. For proper diagnosis, the food intake behaviour of the patients has to be monitored. We developed an approach to automatically analyze food intake using chewing and swallowing sounds. Two microphones of a sensor system in the outer ear canal and above the ear record these sounds. A central signal processing element is the food intake activity detection. It detects periods of eating and drinking activity and rejects sounds not related with food intake. An artificial neural network in feed-forward structure is used as main part of the food intake activity detection algorithm. It is trained on a small data set containing chewing and swallowing sounds, speech and environmental sounds. Input features were computed from time and frequency domain. The features were computed for the signals of the in-ear microphone and the reference microphone, respectively. A data set of food intake sounds and various environmental sounds of everyday life is used as test set for evaluation. We compared the results with those of a simple detection algorithm based on signal energy ratio. While the simple detection algorithm failed in many cases of environmental sounds, the artificial neural network classifier could strongly reduce the number of false positive detections. It provides a basic tool to analyze temporal patterns of human food intake behaviour. In cases of food intake monitoring, the algorithm can be used to trigger food classification and bite weight estimation at periods of real food intake.
\end{abstract}

\section{Introduction}

According to the World Health Organization (WHO), the number of nutrition-related diseases like obesity and Diabetes mellitus increased significantly in the past decades [1]. Therefore, food intake monitoring gains more impact today. A precise timesaving protocol method for eating behaviour is needed. Monitoring of food consumption is often done manually using questionnaires today. This method suffers from the disadvantages of being timeconsuming and of low reliability. According to Schoeller [2], underestimation of the amount of consumed food of 50 $\%$ is not unusual. Many approaches have been made to ease food intake monitoring by recording and evaluation of food intake sounds [3-5]. These approaches are expected to be of great benefit for the user.

However, recording and analysis of food intake has to be triggered to real eating and drinking periods. Otherwise computing will be of high effort and large amounts of memory will be needed. In this paper, we present a method to detect periods of food intake in raw sound data using an artificial neural network (ANN).

This paper is organized as follows: Section 2 gives a brief description of our sensor system. The work of the food intake activity detection algorithm using an ANN is presented in this section, too. Section 3 introduces the evaluation method and presents its results using the ANN. They were compared to those of a simple detection algorithm from our previous work [6]. The conclusion is drawn in Section 4.

\section{Methods}

We built up a novel sensor system with two miniaturized electret microphones integrated in a hearing aid package (Phonak SuperFont) [7]. Hearing aid packages were chosen to address elderly people under conditions of ambient assisted living or retirement homes. This choice was made because problems with obesity and overweight are even worse within the population of elderly people. According to the federal statistical office of Germany [8], $72 \%$ of men and $59 \%$ of women aged 60 years and above in Germany have been overweight or obese in 2009. In our investigation, food intake sound data were recorded by the in-ear microphone applied in the outer ear canal. The reference microphone just above the

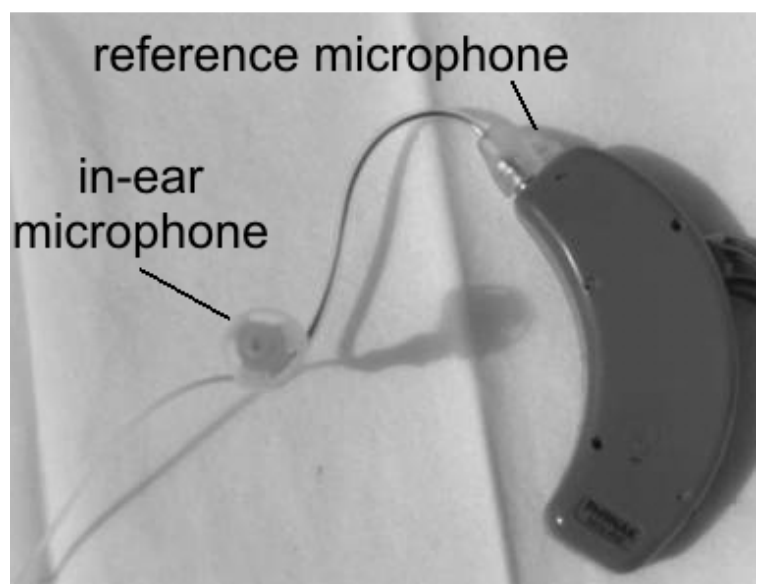

Figure 1 Hearing aid package with both integrated microphones 
outer ear took environmental sound data. Sampling rate is $11,025 \mathrm{~Hz}$ at a resolution is $16 \mathrm{bit}$. Image 1 shows the sensor system.

We recorded data of 51 participants aged 15 to 77 years (mean: 34.8 years) with natural dentition. They were instructed to eat ten pieces of seven types of food (potato chip, peanut, walnut, carrot, apple, chocolate, pudding), respectively, and to drink 30 sips of a drink of their choice (juice or water). Selection of these types of food was made due to practical reasons and in order to use some of the most common snacks. Records were done in a quiet recording room or office room.

Additional records containing environmental sounds were taken from a subset of 4 of these participants. They contained speech of reading, utterance of a constant vowel ' $a$ ' over 15 seconds, coughing, walking around in a room, scratching one's own head, drumming with the fingers on the desk and clanging a spoon on an empty ceramic cup.

We used a subset of 4 participants consuming the presented types of food to represent food intake sounds. This limitation was made to keep the balance of the amount of food intake sounds and environmental sounds. Otherwise, the amount of food intake sounds would have been overrepresented. Hence, an overall evaluation would not represent the ability to reject environmental sounds correctly.

Further records on other types of food and environmental sounds were taken from a single participant and used as training data for the detection algorithms. All records were labelled manually distinguishing between chewing sounds, swallowing sounds, sounds not related with food intake (speech, noise, etc.) and silent periods without any sound. In our previous work [6], we presented a simple algorithm for food intake activity detection. It used different signal energy levels of recorded food intake sounds by both microphones. Being tested on different records of sounds not generated by food intake, this simple algorithm failed in several cases. Especially on records of scratching the own head, clanging tableware or loud reading, many sounds were falsely detected as food intake sounds. We wanted to overcome this problem by training an ANN on records of food intake and environmental sounds. In the training step, environmental sounds with high detection error rates could be included to enhance the activity detection performance. Training and testing the ANN was done off-line using the Neural Network Toolbox of Matlab R2008a (The Mathworks Inc). A two-layer feed-forward backpropagation network was used as ANN structure for this task. The hyperbolic tangent sigmoid function was the transfer function of the hidden layer. This layer consisted of 20 neurons. We implemented two outputs, $\mathrm{y}_{1}$ and $\mathrm{y}_{2}$, one to designate food intake sounds and the second one to designate sounds not related with food intake. A linear transfer function was used for the two neurons of the output layer. Input features were computed from non-overlapping frames of 4096 samples (i.e. $372 \mathrm{~ms}$ ) of the record. Input features were:

- the smoothed ratio $\mathrm{R}$ of signal energy equivalents of both microphones of the sensor system (as in [6]):

$$
R=\frac{S_{\text {in-ear }}}{S_{\text {reference }}},
$$

where $S_{\text {in-ear }}$ and $S_{\text {reference }}$ are the sums of absolute signal amplitude values of the in-ear record and the reference record, respectively,

- signal variances var from the records of each microphone and their smoothed ratio, where signal variance is calculated as

$$
\operatorname{var}=\frac{1}{k} \sum_{k}\left(x(k)^{2}-\bar{x}^{2}\right),
$$

where $\mathrm{k}$ is the index of the samples, $\bar{x}$ is the mean signal value and $\mathrm{x}$ is the signal value,

- zero crossing rate from the records of each microphone and their smoothed ratio,

- centroid frequency $\mu_{\text {cent }}$ from the records of each microphone and their ratio. Centroid frequency is calculated as:

$$
\mu_{\text {cent }}=\frac{\sum_{f} f \cdot \operatorname{ampl}(f)}{\sum_{f} \operatorname{ampl}(f)},
$$

where $f$ is the frequency and $\operatorname{ampl}(f)$ is the corresponding absolute spectral value of the spectrum calculated by using the FFT on the current signal frame.

- maximum signal value from the current frame of the records of each microphone and their smoothed ratio and

- spectral variance var $_{\text {spect }}$ from the records of each microphone and their smoothed ratio. The spectral variance is calculated as:

$$
\operatorname{var}_{\text {spect }}=\frac{1}{f} \sum_{f}\left(\operatorname{ampl}(f)^{2}-\overline{a m p l}^{2}\right),
$$

where $\mathrm{f}$ is the frequency, $\overline{a m p l}^{2}$ is the mean absolute spectral value and ampl(f) is the absolute spectral value corresponding to $f$ of the spectrum calculated by using the FFT on the current signal frame.

Smoothing of any of these ratios $\mathrm{R}_{\mathrm{v}}$ is done as follows:

$$
\rho_{v}(l)=0.9 \cdot \rho_{v}(l-1)+0.1 \cdot R_{v},
$$

where 1 is the index of the current signal frame, $\rho$ is the smoothed ratio and $v$ is an index for any of the features described above. Overall, 16 features are computed per frame and used as input for the ANN.

Training is done based on labelled records. The output reference for training was computed based on the manual annotation of the records. Frames with a predominant number of samples labelled as chewing or swallowing sounds were counted as food intake activity. Analogously, frames were not marked as food intake activity if a predominant number of their samples correspond to labels of environmental sounds or silence. Excluded from this were silence periods with durations of less than one second. This was done because short silence durations can occur both in periods of food intake between the chewing strokes and in periods of environmental sounds (e.g. between two utter- 
ances). Training data were records of one participant eating a piece of raw carrot, consuming a bite of potato croquette, rustling with a plastic bag, speaking and clattering a bunch of keys. Cumulated, 105 seconds of sound data were contained in the test records.

The trained ANN was used for detection of food intake activity in sound records. Food intake activity was marked as detected if the following condition was fulfilled:

$$
y_{2}<\lambda \cdot y_{1} \text {. }
$$

$\lambda$ is a threshold parameter for the detection algorithm.

\section{$3 \quad$ Results}

The ANN was trained with backpropagation network training using the training data described above. Evaluation was not done on these training data. Both food intake activity detection algorithms (the ANN and the one from [6]) were evaluated on parts of the recorded sounds, as described in section 2. Overall, the test set consisted of 92 min of food intake and 83 min of environmental sounds.

The automated detection results were compared to the label files of the corresponding record. Positive food intake activity detection was evaluated as true positive (TP) for signal samples whose labels belong to chewing or swallowing sound classes. Otherwise, it was evaluated as false positive (FP). Automated rejection of food intake activity was evaluated as true negative (TN) for signal samples whose labels belong to silence periods or periods of sound not related to food intake. Otherwise, it was evaluated as false negative (FN). Silence periods of durations less than one second were excluded from this evaluation. We calculated accuracy, precision and recall as follows:

$$
\begin{aligned}
\text { accuracy } & =\frac{\sum_{i}(T P+T N)(i)}{\sum_{i}(T P+T N+F P+F N)(i)}, \\
\text { precision } & =\frac{\sum_{i} T P(i)}{\sum_{i}(T P+F P)(i)}
\end{aligned}
$$

and

$$
\text { recall }=\frac{\sum_{i} T P(i)}{\sum_{i}(T P+F N)(i)}
$$

where $\mathrm{i}$ is the index of the current record.

Receiver Operating Characteristic (ROC) graphs were generated by varying $\lambda$ in the detection condition (6) of the algorithm and in the detection condition of the simple algorithm presented in [6]. Image 2 shows the ROC graphs of both food intake activity detection algorithms.

It can be seen that varying $\lambda$ in the simple food intake activity detection algorithm resulted in remarkable recall values over $95 \%$ at a precision of $70 \%$. However, varying the threshold parameter did not result in precision values above $85 \%$. This shows that the simple algorithm has

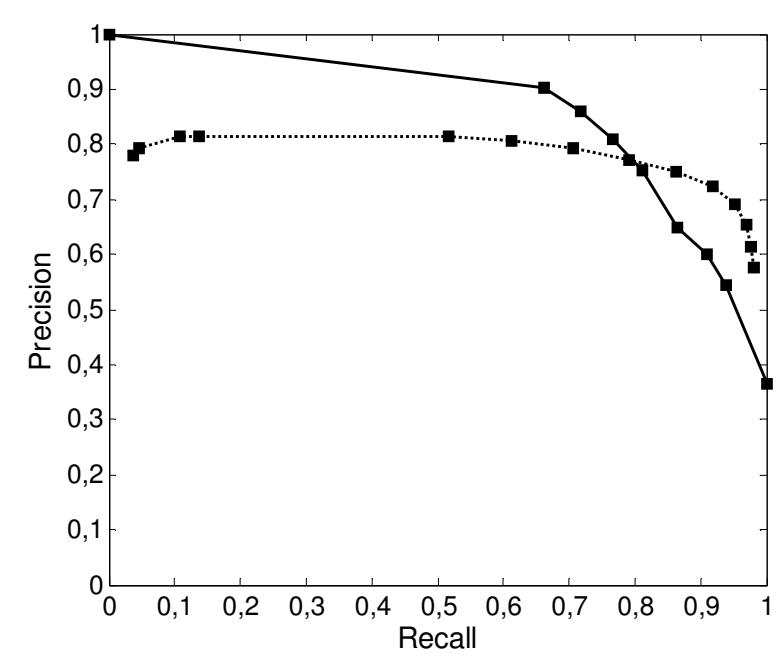

Figure 2 ROC graphs of the food intake activity detection algorithm using an ANN (solid line) and the algorithm from [5] (dotted line). Best performance is found towards the top-right corner (high precision and recall).

large rates of false positive detections, especially in cases of high amplitudes of environmental sounds. Food intake activity detection using an ANN works at a precision of 90 $\%$, but achieves recall values of $66 \%$. In this case, the number of false positive detections can be decreased at the cost of food intake period detections. Best detection results in terms of precision and recall were $85 \%$ accuracy, $86 \%$ recall and $72 \%$ precision for food intake activity detection

Table 1 Average detection accuracies of both algorithms on food intake sounds in percent and their absolute difference

\begin{tabular}{c|ccc} 
Food Sound & with ANN & algorithm [5] & difference \\
\hline potato chips & 78.6 & 87.4 & -8.8 \\
peanut & 77.4 & 87.0 & -9.6 \\
walnut & 73.9 & 89.3 & -15.4 \\
carrot & 72.3 & 90.9 & -18.7 \\
apple & 78.0 & 93.6 & -15.6 \\
chocolate & 63.7 & 82.8 & -19.1 \\
pudding & 72.9 & 82.7 & -9.8 \\
water & 74.7 & 78.8 & -4.1
\end{tabular}

\begin{tabular}{|c|c|c|c|}
\hline $\begin{array}{c}\text { Environmental } \\
\text { Sound }\end{array}$ & with ANN & algorithm [5] & difference \\
\hline reading & 99.7 & 73.6 & 26.1 \\
\hline vowel & 99.8 & 100.0 & -0.2 \\
\hline walking & 97.7 & 88.6 & 9.1 \\
\hline $\begin{array}{l}\text { drumming } \\
\text { finger }\end{array}$ & 99.5 & 99.1 & 0.4 \\
\hline $\begin{array}{l}\text { clanging } \\
\text { spoon }\end{array}$ & 98.8 & 41.2 & 57.6 \\
\hline coughing & 86.7 & 89.7 & -3.1 \\
\hline $\begin{array}{l}\text { scratching } \\
\text { head }\end{array}$ & 91.6 & 74.0 & 17.7 \\
\hline
\end{tabular}

Table 2 Average detection accuracies of both algorithms on environmental sounds in percent and their absolute difference 
using the ANN. The simple detection algorithm achieved its best performance values at $84 \%$ accuracy, $72 \%$ recall and $92 \%$ precision. (Best performance values are the values with maximum product of precision and recall.

Table 1 and Table 2 show the average detection accuracies achieved by the use of both algorithms on records of food intake sounds and environmental sounds, respectively. It can be seen in Table 1 that detection accuracies in records of soft food (chocolate, pudding) or water is lower than in records of hard food. This is due to the low sound amplitude in these records. Using the ANN instead of the algorithm from [6] decreased the detection accuracies (negative differences). Comparable detection accuracies were achieved for soft and hard texture food. Table 2 shows only moderate performance of the algorithm from [6] at rejecting sounds of reading, clanging a spoon at an empty cup and scratching the own head. This is a problem in practical use, for all these sounds can occur commonly during eating a meal as well as in periods without food intake. A proper food intake activity detection algorithm has to reject these sounds. The detection algorithm based on the ANN showed high performance at rejecting these sounds. Therefore, it gains practical importance.

In their work [3], Amft et al. received both precision and recall values of $98 \%$ by the use of models based on spectral band energies and information of cepstral coefficients, zero crossings and amplitude fluctuation. These remarkable results were achieved with a sensor setup, where foam occluded the outer ear canal and dampened environmental sounds. This reduced the natural hearing ability of the user at this ear. Shuzo et al. [5] received recognition accuracies of $80 \%$ in the task of separating eating hard food, eating soft food, drinking and speaking. However, a direct comparison to food intake activity detection was not possible because there was no separation of the detection and the classification task.

\section{Conclusion}

There is a strong need to analyze human food intake patterns to overcome the trend of increasing numbers of obese persons in our civilization. Automated methods for food intake activity detection present a cost-effective and promising way to help people to do this task.

We investigated the use of artificial neural networks on sound data of food intake and environmental sound to detect food intake activity. The ANN achieved promising results in detecting environmental sounds. The benefit of using an ANN is the possibility for adaptation to special kinds of disturbing sounds. Using example records of common sounds from the environment of the user, an ANN with high precision and recall can be achieved for these living conditions. The simple food intake activity detection algorithm presented in [6] can only be adapted in a very restricted way.

The signals used in this study represented a part of typical sounds from eating and from possible environmental disturbances. However, they were only a small set of the whole variety of possible sounds. Recordings from more types of food and several other environmental sounds will be taken. The performance of the sensor system will be investigated on these recordings to verify the algorithm.

In the present study, we did not investigate the influence of quasi-stationary background noise on the detection performance. For the application in ambient assisted living or retirement homes, this simplification can be made because of the low level of background noise in these institutions (compared to e.g. university restaurants). However, for less restricted use, the system will be investigated under different noise levels.

The presented method is seen as an important step towards the long-time analysis of human food intake patterns. The algorithm can be used to detect time and duration of food intake. In combination with classification algorithms, it can furthermore reduce the amount of sound data to classify by enabling the classification only for data with detected food intake activity. Hence, only periods of real food intake will be used for estimation of the type of consumed food or the bite weight. Further work will address long time studies for validation of the presented method in dayto-day living.

\section{References}

[1] World Health Organization: Obesity and overweight: What are overweight and obesity? Fact sheet $\mathrm{N}^{\circ} 311$. Geneva: WHO, 2006

[2] Schoeller D.A.: Limitations in the assessment of dietary energy intake by self-report. Metabolism 44, pp. 18-22, 1995

[3] Amft, O., Stäger, M., Lukowicz, P. and Tröster, G.: Analysis of chewing sounds for dietary monitoring. Proceedings of 7th International Conference on Ubiquitous Computing - UbiComp 2005 (Tokyo, Japan, September 2005) pp. 56-72, 2005

[4] Lopez-Meyer, P., Schuckers, S., Makeyev, O. and Sazonov, E.: Detection of periods of food intake using Support Vector Machines. Proceedings of 32nd Annual International Conference of the IEEE EMBS (Buenos Aires, Argentinia, 31 August - 4 September 2010), pp. 1004-1007, 2010

[5] Shuzo, M. et al.: Wearable eating habit sensing system using internal body sound. Journal of Advanced Mechanical Design, Systems and Manufacturing 4(1), pp. 158-166, 2010

[6] Päßler, S., Fischer, W.-J.: Food Intake Activity Detection Using a Wearable Microphone System. Proceedings of the Seventh International Conference on Intelligent Environments - IE'11 (Nottingham, UK, 25-28 July 2011) pp. 298-301, 2011

[7] Päßler, S., Wolff, M., Fischer, W.-J.: Food intake monitoring: An acoustical approach to automated food intake activity detection and classification of consumed food. Physiol. Meas. 33, pp. 1073-1093, 2012

[8] Statistisches Bundesamt: Gesundheit im Alter (Wiesbaden, Germany) URL: https://www.destatis.de, 2011 